\title{
Uso de colgajos musculares en las perforaciones traumáticas del esófago*
}

\author{
Drs. RAFAEL ANDRADE-ALEGRE ${ }^{1}$, CARLOS JIMÉNEZ ${ }^{1}$, ENRIQUE LIAO GUEVARA ${ }^{1}$
}

1 Sección de Cirugía Torácica, Hospital Santo Tomás. Panamá, República de Panamá.

\begin{abstract}
Use of muscular flaps to repair traumatic esophageal perforations. Report of two cases
\end{abstract}

The management of esophageal perforations is variable, ranging from conservative management to esophageal excision. Muscular flaps are used specially when there are associated lesions in trachea or aorta. We report two patients managed using this last technique. A 31 years old woman that received a gun shot in the neck that was operated, repairing two tracheal and two esophageal perforations. The patient had to be reoperated 28 days later, due to a dehiscence of the esophageal suture. A muscular flap was used to repair the lesion with a good postoperative evolution. A 17 years old male that received a gunshot in the left supraclavicular region. Eight days later, he was operated, draining a right empyema and repairing an esophageal perforation using a muscular flap. The patient had a good postoperative evolution.

Key words: Traumatic esophageal perforation, primary repair, muscle flap, delayed diagnosis.

\section{Resumen}

Las perforaciones del esófago, especialmente las de manejo tardío, representan un dilema y un reto para el cirujano. El manejo de las perforaciones tardías ha sido controversial y las opciones terapéuticas incluyen desde manejo conservador y simple drenaje hasta la resección del esófago. Se presentan 2 casos de perforaciones esofágicas producidas por proyectil de arma de fuego referidos a los 28 y 8 días respectivamente. En ambos se realizó reparación primaria reforzada con colgajo muscular tardío.

Palabras clave: Perforación traumática del esófago, cierre primario, colgajo muscular, diagnóstico

\section{Introducción}

En las perforaciones traumáticas del esófago de manejo temprano (menos de 24 horas de evolución) no hay mayor controversia y el tratamiento consiste en cierre primario. Los colgajos musculares en este grupo de lesiones pueden ser utilizados a dis- creción del cirujano o en casos donde hay lesiones asociadas como la tráquea ${ }^{1}$ o la arteria carótida ${ }^{2}$. En las perforaciones de manejo tardío (más de 24 horas de evolución) hay numerosas técnicas y opciones quirúrgicas ${ }^{3-6}$. A continuación describimos dos casos de uso de colgajo muscular pediculado de músculo esternocleidomastoideo e intercostal

\footnotetext{
*Recibido el 2 de Diciembre de 2008 y aceptado para publicación el 11 de Febrero de 2009.

Correspondencia: Dr. Rafael Andrade-Alegre

Apdo. Postal 0832-01450, Panamá, República de Panamá. Fax: (507) 229-6646

E-mail: toravasc@cwpanama.net
} 
respectivamente para reforzar la reparación de perforaciones traumáticas del esófago producidas por proyectil de arma de fuego (PAF).

\section{Caso clínico 1}

Mujer de 31 años quien recibió herida por PAF en el cuello. Manejada inicialmente por el Servicio de Cirugía General y Otorrinolaringología. Durante la cirugía se repararon 2 perforaciones traqueales y 2 de esófago cervical. Además se realizó traqueostomía y se dejó drenaje de Penrose. A partir del cuarto día post-operatorio presenta salida de contenido esofágico por el drenaje y se opta por manejo conservador omitiendo la vía oral e iniciando nutrición parenteral total. El drenaje aumentó progresivamente hasta $500 \mathrm{ml}$ por día (Figura 1). Es referida a nuestro cargo a los 28 días y es llevada al quirófano donde se realizó exploración cervical. Se encontró dehiscencia de la línea de sutura en la pared posterior del esófago parcialmente adherida a la fascia prevertebral. Una vez movilizado el esófago es evidente dehiscencia total de la reparación inicial (Figura 2). Se procede a suturar el defecto con puntos totales de seda 3-0, teniendo particular cuidado en incluir la mucosa de los extremos de la herida. Seguidamente se coloca colgajo pediculado del vientre interno del músculo esternocleidomastoideo sobre la línea de sutura fijándose con puntos de seda 3-0 (Figura 3). La evolución fue satisfactoria, con pequeña fístula residual que cerró espontáneamente. En seguimiento a 8 meses, la paciente tolera dieta corriente y no hay evidencia de estenosis.

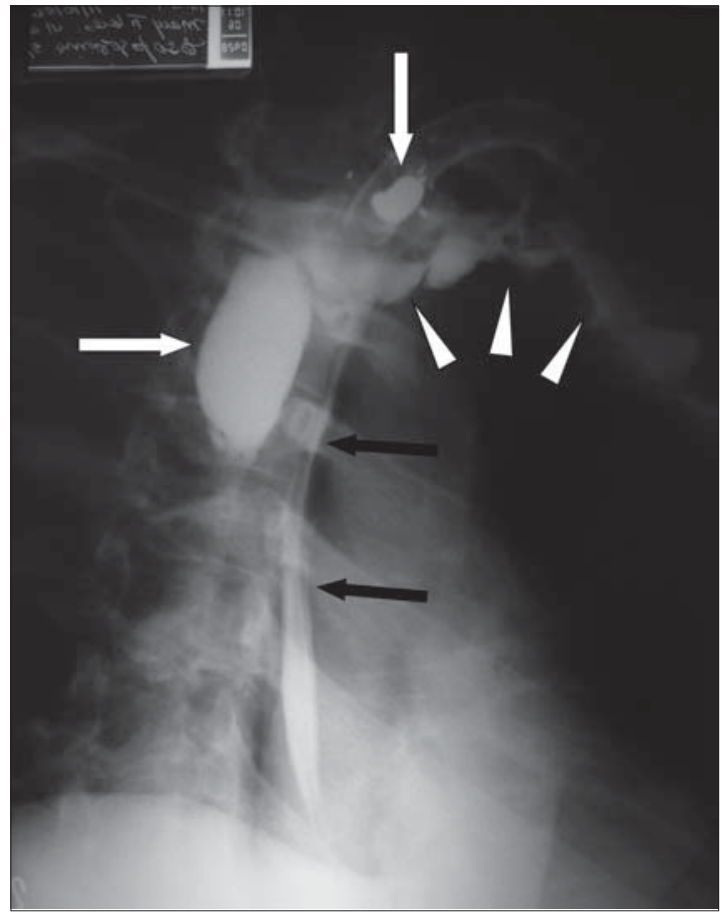

Figura 1. Esofagograma. La flecha blanca horizontal muestra colección de fondo ciego del medio de contraste a nivel del cuello y horquilla esternal. La flecha blanca perpendicular señala el PAF. Las flechas negras muestran el esófago. Los triángulos blancos evidencian el gran escape del medio de contraste.
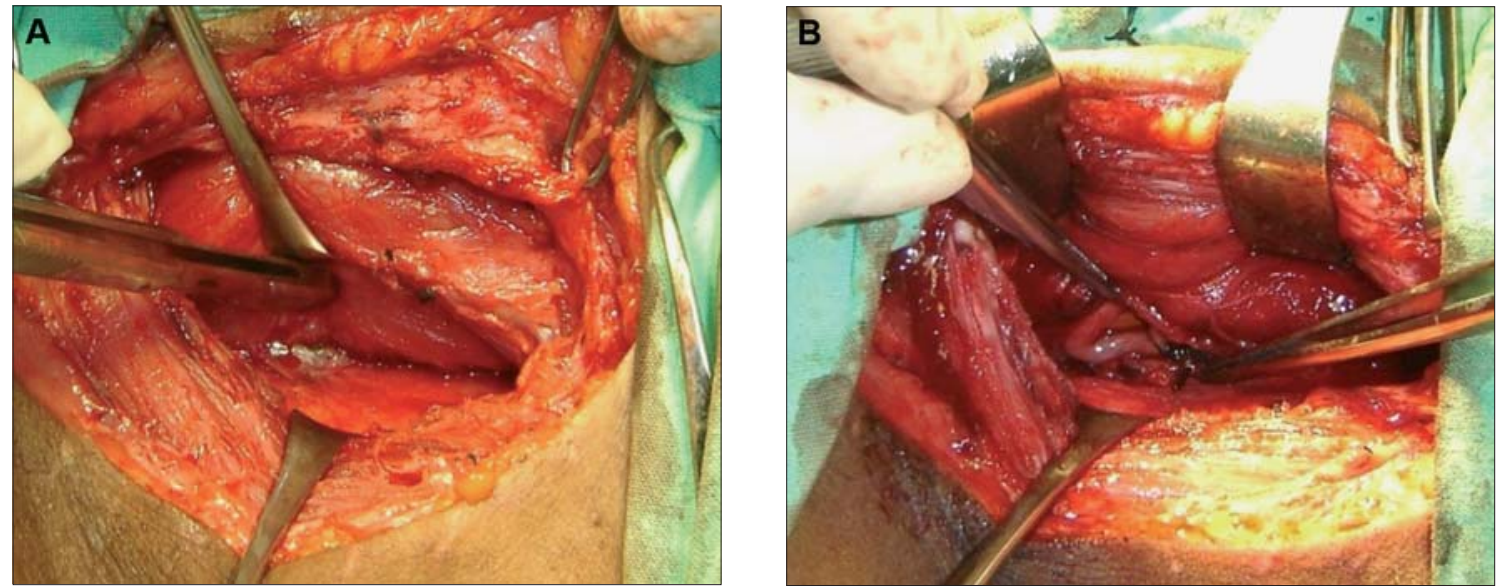

Figura 2. A) Perforación del esófago posterior adherida parcialmente a la fascia prevertebral. B) Dehiscencia total de la herida. Se observan los puntos de la sutura previa. 


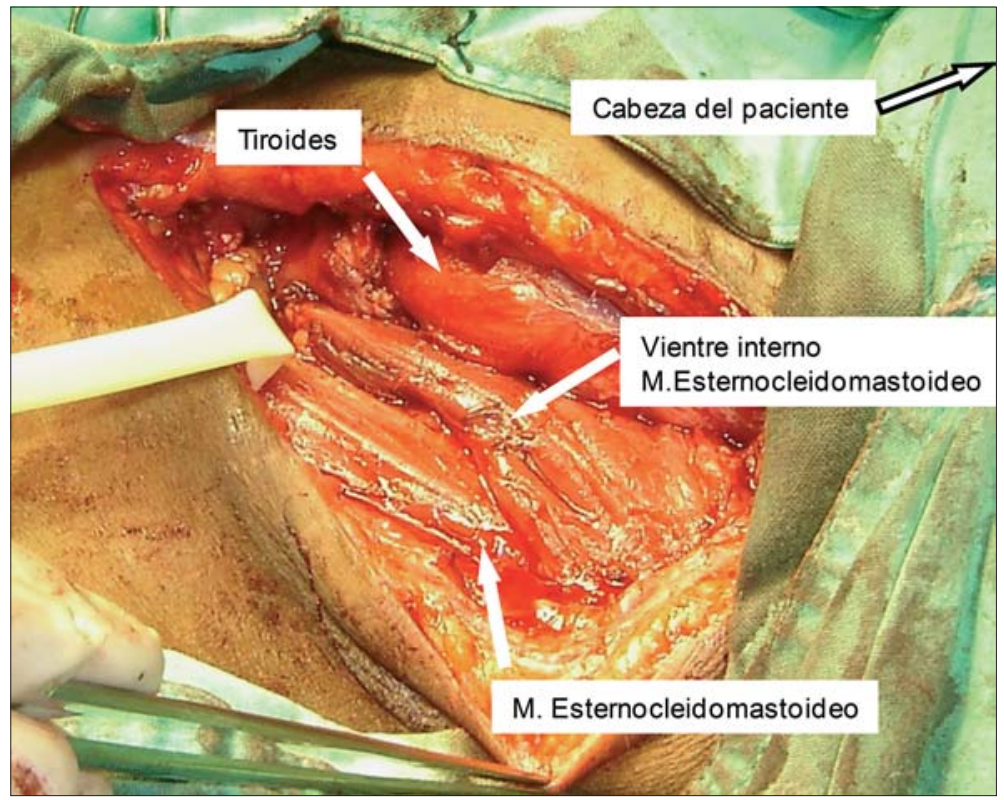

Figura 3. Colgajo pediculado de vientre interno del músculo esternocleidomastoideo reforzando la línea de sutura.

\section{Caso clínico 2}

Varón de 17 años quien sufre herida por PAF en región supraclavicular izquierda. Manejado inicialmente en hospital de segundo nivel. Se diagnosticó hemotórax derecho, por lo que se colocó tubo pleural drenando inicialmente moderada cantidad de sangre. El paciente tuvo una evolución tórpida con afectación del estado general. Es entonces cuando se sospecha lesión esofágica y se administra azul de metileno evidenciándose escape del material a través del tubo pleural. El paciente es referido a la Sección de Cirugía Torácica del Hospital Santo Tomás 8 días después de haber sufrido la herida (Figura 4). Requirió del uso de inotrópicos y fue necesario someterlo a ventilación mecánica por shock séptico. Es llevado al quirófano donde se efectúa esofagoscopia rígida encontrándose perforación esofágica a unos $27 \mathrm{cms}$ de la arcada dentaria. Seguidamente se realiza toracotomía posterolateral derecha encontrándose empiema y engrosamiento pleural. El empiema es evacuado y el pulmón decorticado. Luego se expone el tercio medio del esófago encontrándose perforación posterior de aproximadamente $1 \mathrm{~cm}$. La perforación es suturada con puntos totales de seda 3-0, teniendo cuidado de incluir completamente la mucosa involucrada. Se diseca el músculo intercostal del espacio costal donde se realizó la incisión y se coloca colgajo muscular sobre la línea de sutura y se fija con puntos de seda 3-0. Se realizó yeyunostomía para alimentación. El paciente tuvo un posoperatorio sin complicaciones. El esofagograma de control (Figura 5) no mostró escape del medio de contraste y fue dado de alta al $10^{\circ}$ día. En seguimiento a 6 meses el paciente tolera dieta corriente sin evidencia de estenosis.

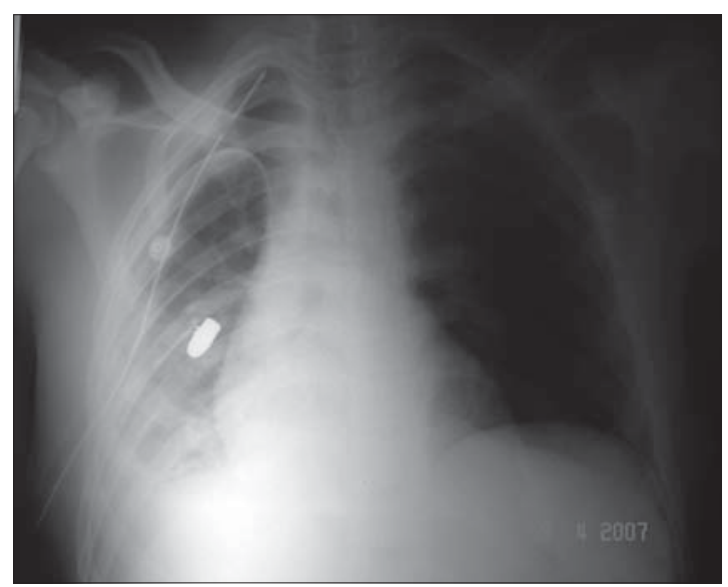

Figura 4. Radiografía de tórax. Hidroneumotórax y engrosamiento pleural. Se aprecia PAF sobre campo pulmonar derecho. 


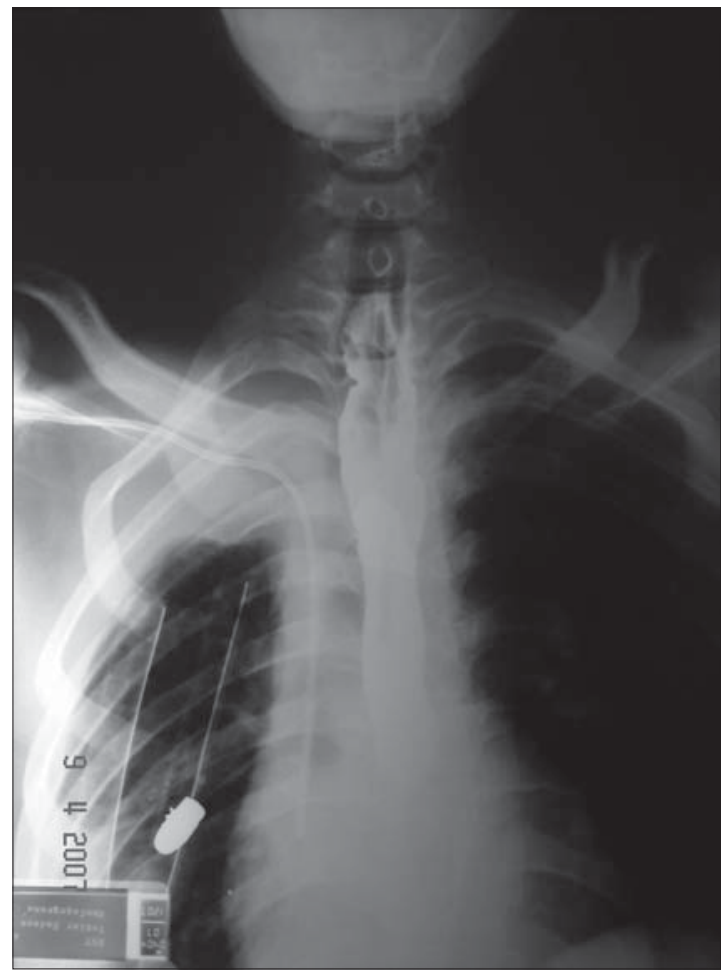

Figura 5. Esofagograma al $5^{\circ}$ día posoperatorio. Nótese que no hay escape del medio de contraste.

\section{Discusión}

Las perforaciones traumáticas del esófago de manejo tardío son controversiales y esto es evidente por las múltiples opciones terapéuticas descritas. En estos casos hay incidencia elevada de escapes de la línea de sutura debido al severo proceso inflamatorio que se produce ${ }^{7}$. Por esta razón algunos autores proponen individualizar el tratamiento de los pacientes en estas condiciones ${ }^{5,8}$. La sutura primaria con la realización de algún tipo de colgajo para reforzarla es una de las técnicas que ha tenido mejores resultados en los últimos años y es recomendada como tratamiento de elección en cualquier tipo de perforación por varios autores ${ }^{7,9,10}$. En nuestro primer caso, el esófago fue reparado con puntos separados en un solo plano y la línea de sutura se reforzó con músculo esternocleido- mastoideo; en el segundo paciente, la línea de sutura también fue reforzada, en esta ocasión se usó músculo intercostal. La evolución fue satisfactoria para los dos pacientes, sin evidencia de estenosis.

Si bien individualizamos el tratamiento de nuestros pacientes con perforaciones del esófago con tiempos de evolución mayor de 24 horas, vemos en el uso de los colgajos musculares una opción segura y con buenos resultados. Cuando se opta por cierre primario en perforaciones del esófago de manejo tardío, parece recomendable reforzar la línea de sutura con colgajo muscular.

\section{Referencias}

1. Feliciano DV, Bitondo CG, Mattox KL, Romo T, Burch JM, Beall AC. Combined tracheosopageal injuries. Am J Surg 1985; 150: 710-715.

2. Losken A, Rozycki GS, Feliciano DV. The use of the sternocleidomastoid muscle flap in combined injuries to the esophagus and carotid artery or trachea. J Trauma 2000; 48: 815-817.

3. Kiev J, Amandola M, Bouhaidar D, Sandhu BS, Zhao $\mathrm{X}$, Maher J. A management algorithm for esophageal perforation. Am J Surg 2007; 194: 103-106.

4. Brinster CJ, Sunil S, Lawrence L, Marshall MB, Kaiser LR, Kucharczuk JC. Evolving options in the management of esophageal perforation. Ann Thorac Surg 2004; 77: 1475-1483.

5. Ríos Zambudio A, Martínez de Haro LF, Ortiz Escandell MA, Durán H, Munitiz Ruiz V, Parrilla Paricio P. Perforaciones esofágicas. Presentación de 23 casos. Gastroenterol Hepatol 2000; 23: 379-383.

6. Andrade-Alegre R. T-tube intubation in the management of late traumatic esophageal perforations: case report. J Trauma 1994; 37: 131-132.

7. Richardson JD. Management of esophageal perforations: the value of aggressive surgical treatment. Am J Surg 2005; 190: 161-165.

8. Andrade-Alegre R. Surgical treatment of traumatic esophageal perforations. Analysis of 10 cases. Clinics 2005; 60: 375-380.

9. Gouge TH, Depan HJ, Spencer FC. Experience with the Grillo pleural wrap procedure in 18 patients with perforation of the thoracic esophagus. Ann Surg 1989; 209: 612-619.

10. Pla V, Cuesta MA, van den Broek WT. Tratamiento de las perforaciones del esófago torácico. Cir Esp 2005; 77: $327-331$. 